\title{
Insect galls on Myrtaceae: richness and distribution in brazilian restingas
}

\author{
Valéria Cid Maia ${ }^{1 *}$ (10 \\ ${ }^{1}$ Museu Nacional, Quinta da Boa Vista, 20.940-040, São Cristóvão, RJ, Brasil \\ *Corresponding author: Valéria Cid Maia,e-mail: maiavcid@acd.ufrj.br
}

MAIA, V. C. Insect galls on Myrtaceae: richness and distribution in brazilian restingas. Biota Neotropica. 19(1): e20180526. http://dx.doi.org/10.1590/1676-0611-BN-2018-0526

\begin{abstract}
Inventories in Brazilian restingas have been indicating that Myrtaceae are the plant family with the greatest richness of insect galls. A compilation of published data plus new records was elaborated with the aim of stablishing the number of gall morphotypes on this family in this physiognomy of the Atlantic Forest, producing a list of galled species, pointing out the predominant gall features, evaluating the taxonomical knowledge of the gallers, listing the associated fauna, and based on host plant endemisms and monophagy proposing the endemism of some galling species. Myrtaceae harbor 111 morphotypes of insect gall (about 75\% induced by Cecidomyiidae, Diptera) on 25 host plant species, 15 endemic. Eugenia L. highlights as the plant genus with the highest number of galled species and gall richness. Leaves are the most galled organ. There is a predominance of globoid and fusiform shapes, green color, glabrous surface and a single internal chamber. The taxonomical data on gallers is deficient as many records have been presented at supraspecific levels. The associated fauna is rich and includes parasitoids, inquilines and predators. Twelve species of Cecidomyiidae, a single species of Curculionidae (Coleoptera) and one species of Eriococcidae (Hemiptera) have been associated exclusively with endemic hosts and then are proposed in the present study as endemic too. The geographical distribution of many galls and respective gallers are restricted to the State of Rio de Janeiro, where most inventories have been carried out. For the first time, Eugeniamyia dispar, previously known from a rural area of Rio Grande do Sul and restinga areas of São Paulo, is recorded in the State of Rio de Janeiro.
\end{abstract}

Keywords: Atlantic Forest, diversity, endemism, plant-insect interaction.

\section{Galhas de insetos em Myrtaceae: riqueza e distribuição nas restingas brasileiras}

Resumo: Inventários em restingas brasileiras indicam as Myrtaceae como a família de planta com maior riqueza de galhas de insetos. Uma compilação das informações publicadas acrescida de novos registros foi elaborada com o objetivo de estabelecer o número de morfotipos de galhas, produzir uma lista das espécies botânicas com galhas, indicar as características morfológicas predominantes das galhas, avaliar o conhecimento taxonômico dos galhadores, relacionar a fauna associada, e com base no endemismo das plantas hospedeiras e monofagia, propor o endemismo de algumas espécies galhadoras. As Myrtaceae hospedam 111 morfotipos de galhas de insetos (a maioria induzida por Cecidomyiidae, Diptera) em 25 espécies de plantas, 15 endêmicas. Eugenia L. destaca-se como o gênero botânico com o maior número de espécies hospedeiras e riqueza de galhas. A folha é o principal órgão hospedeiro. Há predomínio das formas globoides e fusiformes, coloração verde, superfície glabra e uma única câmara interna. $\mathrm{O}$ conhecimento taxonômico dos galhadores ainda é deficiente com muitos registros em categorias supraespecíficas. A fauna associada é rica e inclui parasitoides, inquilinos e predadores. Doze espécies de Cecidomyiidae, uma espécie de Curculionidae (Coleoptera) e uma espécie de Eriococcidae (Hemiptera) estão associadas exclusivamente a hospedeiros endêmicos e são propostas neste estudo como endêmicas também. A distribuição geográfica de diversas galhas e seus respectivos galhadores está restrita ao estado do Rio de Janeiro, onde a maioria dos inventários foi realizada. Pela primeira vez, Eugeniamyia díspar (Cecidomyiidae), previamente conhecida de uma área rural do Rio Grande do Sul e de áreas de restingas de São Paulo, é registrada no estado do Rio de Janeiro.

Palavras-chave: Mata Atlântica, diversidade, endemismo, interação inseto-planta. 


\section{Introduction}

Restingas are one of the Atlantic Forest phytophysiognomies, which are defined as long strips of marine sandy depositions, dated of Quaternary (Araújo 1992). In the past, they occupied about $80 \%$ of the Brazilian coast (Lacerda et al. 1993), but due to human activities, especially property speculation, this physiognomy has been suffering a great loss of area. For this reason, restingas are considered one of the most threatened Atlantic Forest physiognomies. Tthe main formations are found from Bahia (Northeast region) to São Paulo (Southeast region) (Neiman 1989).

Restinga vegetation is adapted to extreme environmental conditions, such as high salinity, low water availability and strong sun radiation (Scarano et al. 2001). Nevertheless, this phytophysiognomy harbors a considerable biological diversity and a great variety of unique vegetal communities (Lacerda et al. 1982, Rizzini 1992) due to the topographic diversity of the environmental conditions (Araújo \& Henriques 1984).

Myrtaceae are one of the most species-rich woody plant families in Brazilian restingas (Araújo 2000, Souza \& Morin 2008, Lourenço \& Barbosa 2012), the second most speciose in the Atlantic Forest biome (Stehman et al. 2009), and the fourth in Brazil (Forzza et al. 2010). In fact, this family is highly representative of a variety of biomes of the Neotropics.

The most speciose taxa of Myrtaceae in Brazilian restingas are Eugenia L. and Myrcia DC. (Giaretta \& Peixoto 2015, Martins et al. 2008, Rosario et al. 2005). Both have been also pointed out as the most species-rich genera of Myrtaceae in Brazil with 350 and 260 species, respectively (Landrum \& Kawasaki 1997, Sobral et al. 2018) and in the world with 1,038 and 753 species, respectively (Govaerts et al., 2014).

Several insect gall inventories have been conducted in restinga areas, all in the Southeast region of Brazil, mainly in the state of Rio de Janeiro (Angra dos Reis - Maia \& Oliveira (2010), Mangaratiba - Rodrigues et al. (2014), Rio de Janeiro - Oliveira \& Maia (2005) and Silva \& Maia (2016), Maricá - Maia (2001a), Carapebus - Maia (2001a), Parque Nacional da Restinga de Jurubatiba - Monteiro et al. (2004), Parque Estadual da Costa do Sol - Carvalho-Fernandes et al. (2016), Arraial do Cabo - Monteiro et al. (1994), São João da Barra - Carvalho-Fernandes et al. (2016)), but also in the State of São Paulo (Bertioga - Maia et al. 2008) and Espírito Santo (Guarapari - Bregonci et al. (2010). Besides, additional records were provided by Maia et. al (2002), Silva \& Rodrigues (2011) and Carvalho-Fernandes \& Maia (2011). These inventories have been showing considerable insect gall diversity and highlighting Myrtaceae as the plant family with the greatest number of galled species and the highest gall richness (Table 1). Such richness probably is related to the hygrothermic stress caused by daily temperature contrasts, humity, wind gusts, strong sun radiation and sandy soil (Monteiro et al. 1994, Maia 2001a, Monteiro et al. 2004).

As the available information about insect galls on Myrtaceae are scattered in several publications, the current knowledge is not consolidated. The present review aims to compile all published data on them, add new records and answer the following questions: 1) How many insect galls have been recorded on Myrtaceae in restingas?, 2) How many species of Myrtaceae are galled in this physiognomy?, 3) Which are the galled species?, 4) How is geographic distribution of these plant species and what is their origin and conservational status?, 5) Which are the galled plant organs?, 6) Which are the most frequent gall features (shape, colour, presence or absence of trichomes, number of internal chamber)?, 7) Which are the galling taxa?, 8) How is the geographic distribution of the galling species?, 9) is there any endangered galler?, and 10) How is the composition of predaceous, inquilinous and parasitoids guilds?

\section{Material and Methods}

A bibliographic survey was done to elaborate a list of insect galls on Myrtaceae in restingas. It was carried out by consulting the database Web of Science using "insect gall" and "restinga" as keywords. It included ten published inventories (cited in the Introduction), an insect gall compilation (Maia 2013), scattered records (Maia et al. 2002, Carvalho-Fernandes \& Maia 2011, Silva \& Rodrigues 2011) and two catalogues of Cecidomyiidae (Diptera) of Rio de Janeiro (Maia 2005, Maia \& Barros 2009). Only host plants identified at species level were considered.

Additionally, new records of insect galls are provided. They were obtained from surveys carried out in the Parque Natural Municipal Chico Mendes (Rio de Janeiro, RJ), in February, 2016. The park has 10 paths totaling five quilometers. These paths were pursued in the search of galls for two hours by two persons. The hiking method was adopted to allow the observation of a large number of specimens. Galls were photographed as voucher material.

Botanic names were updated and conservational status of all plant species as well as data on plant endemisms were verified, using the site Flora do Brasil (2020). Based on the high specificity of the gallers in relation to the host plants, galling species associated exclusively with endemic plants were proposed as endemic. Similarly, gallers associated exclusively with threatened plants were proposed as threatened too.

Unfortunately, the morphological gall characterization is not standardized in the literature. The fullest descriptions include galled plant organ, gall shape, color, presence or absence of trichomes, and number of internal chamber, but these features were not equally contemplated in the analysed inventories. Some of them offer very parsimoniosy descriptions. Part of the missing features was retrieved based on gall pictures whenever possible. Besides, different authors adopt different terms to refer to the same gall shape. They are listed in the present study. With regard to the gall morphology, published data are not standardized, resulting in not comparable descriptions as well as in information gaps. Furthermore, different terms have been used to describe the same gall shape. A total of 33 terms were retrieved from literature, but several correspond to the same shape: 1) conical = dropshaped $=$ triangular, 2) lenticular $=$ discoid $=$ epidermic $=$ parenchymal, 3) globoid $=$ globose $=$ globular $=$ spherical $=$ spheroid, 4) cylindrical = tubular, 5) fusiform = elliptical, 6) edge leaf roll $=$ marginal leaf roll, 7) leaf roll $=$ total leaf roll $=$ entire leaf roll, 8) rosette $=$ leaves rosette. In the present study, the following terms were adopted: conical, lenticular, globoid, cylindrical, fusiform, marginal leaf roll, leaf roll, and rosette. Other recorded shapes were claviform, spiraled, ovoid, bivalve, star-shaped, pineapple-shaped, biconical, and leaf blade fold. These last terms were retained in this publication. 
Table 1. Plant families with the highest number of galled species and greatest gall richness in Brazilian restingas.

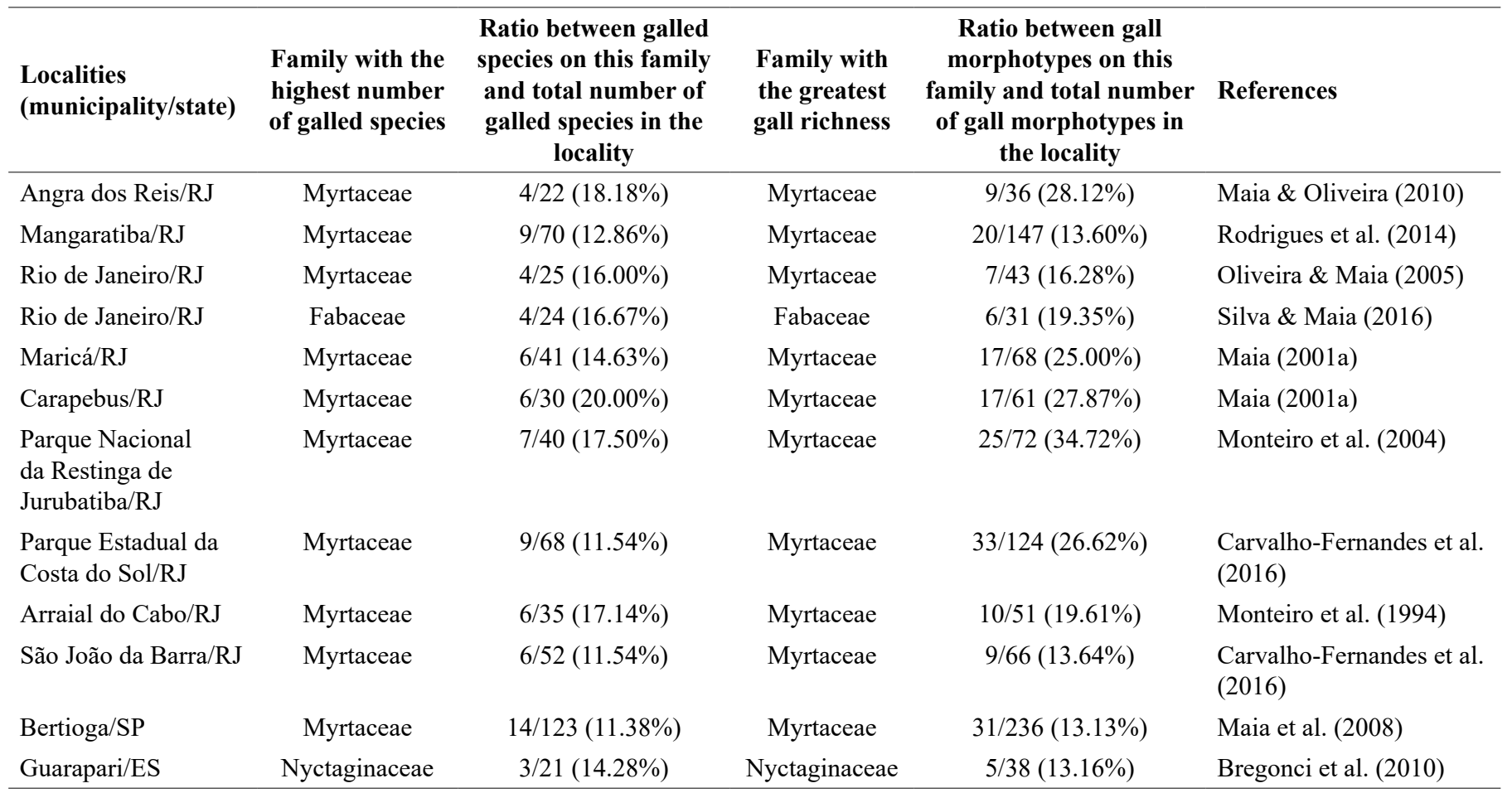

\section{Results}

A total of 111 insect gall morphotypes on 25 plant species of nine genera were accounted (Table 2). This value represents $90.2 \%$ of the gall records on Myrtaceae for restinga, as 12 morphotypes were not included as they were associated with six undetermined plant species. Eugenia L. and Myrcia DC. showed the highest number of galled species (ten each) and the greatest gall richness (47 and 34 morphotypes, respectively). The other genera included a single galled species and from one to ten gall morphotypes. Eugenia and Myrcia together harbored about 73.0\% of the gall richness.

All host plant species are native, being 15 endemic in Brazil. Among the last, 11 occur exclusively in the Atlantic Forest (Table 2). Concerning the conservational status, six plant species are less concerning, 18 have not been yet evaluated, and one is data deficient (Table 2).

Leaves were the most galled plant organ (about 63.0\%), but galls on stems, buds, flowers (flower buds and flower peduncles) and fruits were also recorded (Table 3). The richness of bud and stem galls were similar, about $17.5 \%$ and $14.0 \%$, respectively. Fruit and flower galls were rare (each with less than $3 \%$ ).

The most frequent shapes were conical and globoid (both with 18 morphotypes), fusiform and lenticular (both with 13 morphotypes), and marginal leaf roll (10 morphotypes). The other shapes included from seven to a single morphotype. Green galls predominated $(n=55$, about $49.5 \%$ ), but brown, yellowish, reddish, whitish and black galls were also found. Most galls exhibited a single color, whereas few varied in color. Most galls were glabrous $(n=85$, about $76.6 \%)$ and a single morphotype was hairy $(0.90 \%)$. There is no information about the others. Seventy-four morphotypes were one-chambered (66.67\%), eight were multichambered (7.21\%) and a single one showed from one to three chambers $(0.90 \%)$ (Table 3$)$.
Most gallers were determined ( $\mathrm{n}=80$, about $67.0 \%$ ), but only 21 (about 19.0\%) were identified in species and ten (about 9.0\%) in genus. Records in suprageneric categories predominated, especially at family (Table 3). The gallers were distributed in five orders: Diptera (Cecidomyiidae), Thysanoptera, Hymenoptera, Coleoptera, and Hemiptera. Cecidomyiids induced most galls ( $\mathrm{n}=69$, about $57.0 \%$ ), while the others induced 4 (3.5\%), $3(2.7 \%), 2$ (1.8\%) and $2(1.8 \%)$, respectively. Lepidopteran galls were not found (Table 3 ).

Twelve species of gall midges were identified, being distributed in ten genera, Clinodiplosis Kieffer, 1894, Dasineura Rondani, 1840, Neolasioptera Felt, 1908, Bruggmannia Tavares, 1906, Eugeniamyia Maia, Mendonça \& Romanovski, 1997, Jorgenseniella Maia, 2005, Myrciamyia Maia, 1995, Myrciariamyia Maia, 1994, Neomitranthella Maia, 1996, and Stephomyia Rondani, 1840. Dasineura and Stephomyia were the most speciose genera associated with Myrtaceae in Brazilian restingas. Pacholenus pelliceus Boheman, 1836 (Coleoptera) and Tectococcus ovatus Hempel, 1900 (Hemiptera) were the other identified galling species.

Concerning the geographic distribution, 12 galling species have been recorded, until this moment, only in restingas of Rio de Janeiro State (Table 4). The other species have a less restricted distribution. Eugeniamyia dispar, previously known from a rural area of Rio Grande do Sul and restinga areas of São Paulo, is recorded for the first time in the State of Rio de Janeiro. The distribution of Neolasioptera eugeniae Maia, 1993 includes restinga areas of RJ and an ombrophilous Forest area of Minas Gerais. Dasineura gigantea Angelo \& Maia, 1999 was described from Forest areas of Paraná and Santa Catarina, and later this species was reported in restinga areas of São Paulo. Dasineura myrciariae Maia, 1993 occurs in the states of Rio de Janeiro and Espírito Santo. Jorgenseniella eugeniae Maia, 2005 and Pacholenus 
Maia, V.C. et al.

Table 2. Galled species of Myrtaceae, their synonyms, common names, origin, distribution in Brazilian phytogeographic domains, conservational status and number of gall morphotypes in restingas. DD - deficient data, LC - less concerning, NE - not evaluated, VU - vulnerable.

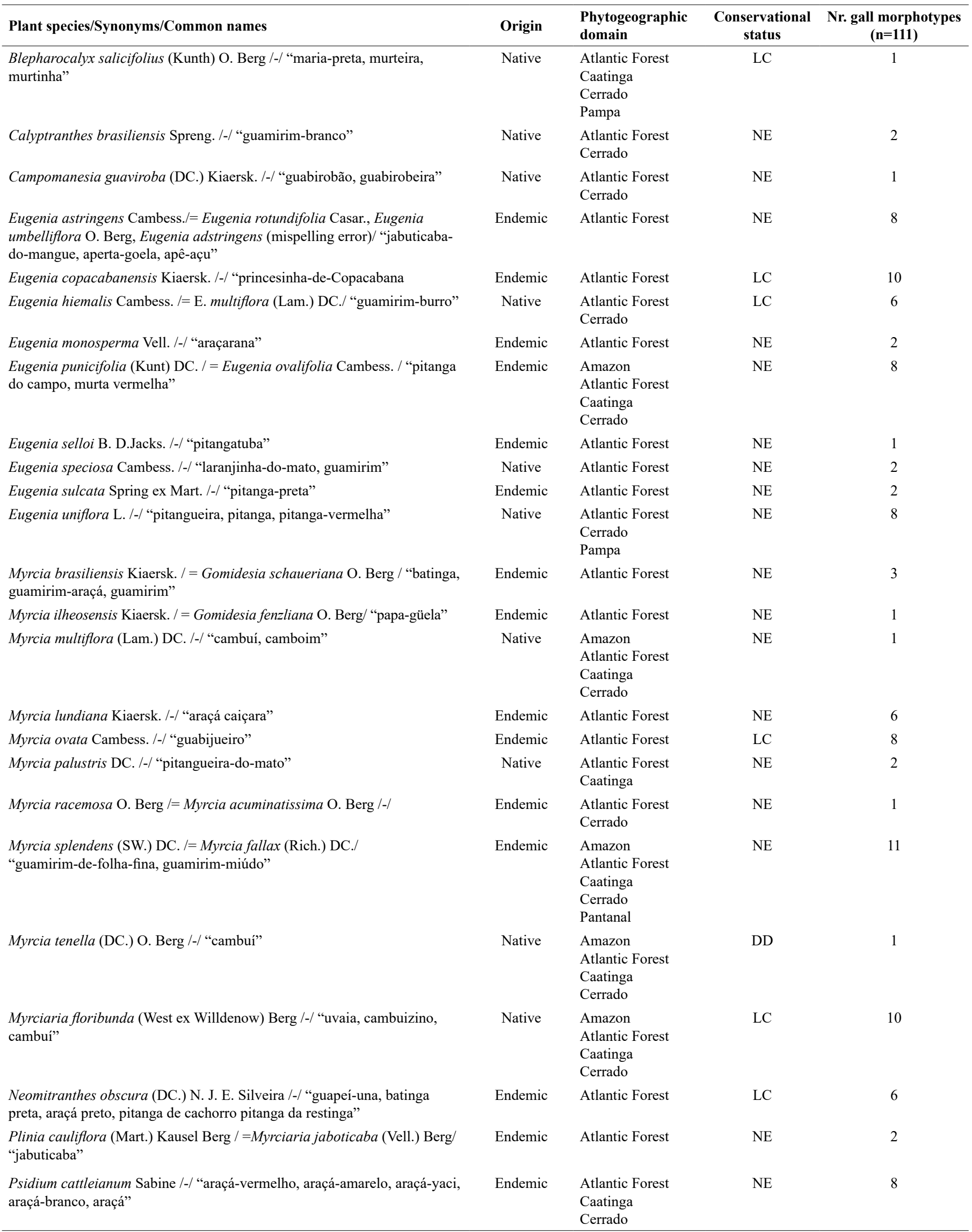


Insect galls on myrtaceae in restingas

Table 3. Gall features on Myrtaceae in Brazilian restingas (shape, color, presence or absence of trichomes, number of internal chamber, galled plant organ, galling order and gallers' taxonomical categories). The total number of gall morphotypes varies due to the available information in the literature. The number of the gall morphotypes by host organ is higher than 111 , because some galls were recorded in two different plant organs.

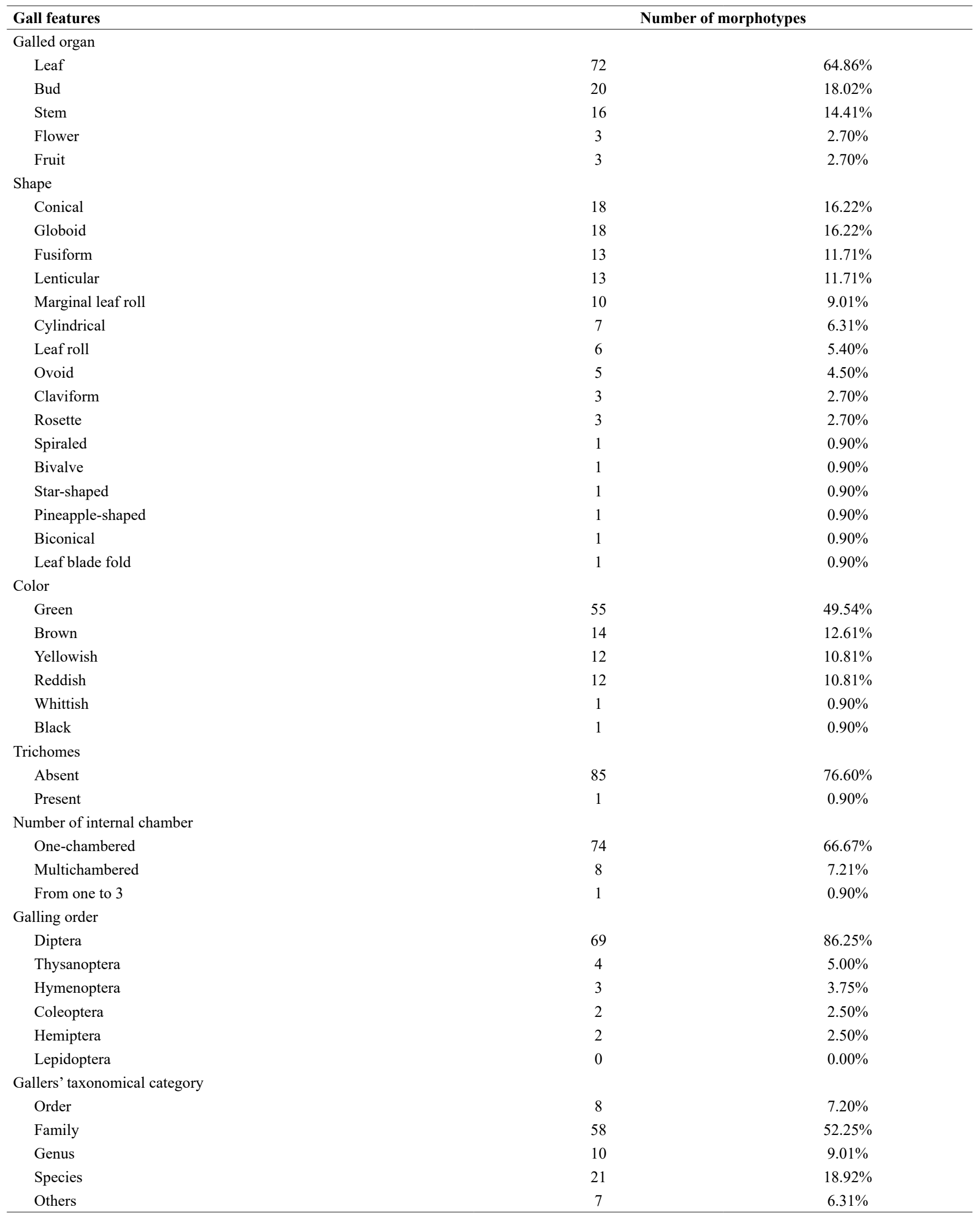


Table 4. Geographical distribution of galling species associated with Myrtaceae in Brazil.

\begin{tabular}{|c|c|}
\hline Galling species & Geographical distribution \\
\hline Bruggmannia sp. & RJ (Arraial do Cabo) \\
\hline Clinodiplosis profusa Maia 2001 & $\begin{array}{l}\text { RJ (Mangaratiba: Ilha da Marambaia; Rio de Janeiro: Grumari; Maricá; Saquarema; } \\
\text { Araruama; Cabo Frio; Arraial do Cabo; Carapebus; Parque Nacional da Restinga de } \\
\text { Jurubatiba; São João da Barra) }\end{array}$ \\
\hline Clinodiplosis sp. & SP (Bertioga) \\
\hline Dasineura copacabanensis Maia, 1993 & RJ (Maricá; Saquarema; Araruama; Cabo Frio; Arraial do Cabo; São João da Barra) \\
\hline Dasineura globosa Maia, 1995 & $\begin{array}{l}\text { RJ (Mangaratiba: Ilha da Marambaia; Rio de Janeiro: Grumari, Niterói; Maricá; } \\
\text { Saquarema; Carapebus; Araruama; Cabo Frio; Arraial do Cabo; Parque Nacional da } \\
\text { Restinga de Jurubatiba, São João da Barra) }\end{array}$ \\
\hline Dasineura sp. 1 & RJ (Maricá) \\
\hline Dasineura sp. 2 & RJ (Maricá) \\
\hline Dasineura sp. 3 & RJ (Maricá, Arraial do Cabo) \\
\hline Dasineura sp. 4 & RJ (Araruama, Arraial do Cabo) \\
\hline $\begin{array}{l}\text { Eugeniamyia dispar Maia, Mendonça \& } \\
\text { Romanovski, } 1997\end{array}$ & $\begin{array}{l}\text { RJ (Rio de Janeiro: Parque Natural Municipal Chico Mendes - new record) } \\
\text { SP (Bertioga) } \\
\text { RS (Porto Alegre) }\end{array}$ \\
\hline Neomitranthella robusta Maia, 1995 & $\begin{array}{l}\text { RJ (Maricá; Saquarema; Araruama; Cabo Frio; Arraial do Cabo; Carapebus; Parque } \\
\text { Nacional da Restinga de Jurubatiba) }\end{array}$ \\
\hline Pacholenus pelliceus Boheman, 1836 & $\begin{array}{l}\text { RJ (Maricá) } \\
\text { SP (Bertioga) }\end{array}$ \\
\hline Stephomyia clavata (Tavares 1920) & $\begin{array}{l}\text { RJ (Carapebus; Parque Nacional da Restinga de Jurubatiba; Araruama; Arraial do Cabo; } \\
\text { São João da Barra) } \\
\text { BA (Madre de Deus) }\end{array}$ \\
\hline Stephomyia espiralis Maia, 1993 & RJ (Mangaratiba: Ilha da Marambaia; Maricá; Araruama; Cabo Frio; Arraial do Cabo) \\
\hline Stephomyia mina Maia, 1993 & RJ (Maricá; Araruama; Arraial do Cabo; Carapebus) \\
\hline Stephomyia rotundifoliorum Maia, 1993 & $\begin{array}{l}\text { RJ (Maricá; Saquarema; Araruama; Cabo Frio; Arraial do Cabo; Carapebus; Parque } \\
\text { Nacional da Restinga de Jurubatiba; São João da Barra) }\end{array}$ \\
\hline Stephomyia tetraloba Maia, 1993 & RJ (Maricá; Arraial do Cabo) \\
\hline Stephomyia sp.1 & RJ (Carapebus) \\
\hline Stephomyia sp.2 & RJ (Mangaratiba: Ilha da Marambaia) \\
\hline Tectococcus ovatus Hempel, 1900 & Wide spread (Brazil, U.S.A. and Japan) \\
\hline
\end{tabular}


pelliceus Boheman, 1836 are known from records in restingas of RJ and SP. Tectococcus ovatus Hempel, 1900 has the widest geographical distribution, including U.S.A. (Florida and Hawaii) and Japan, due to the ornamental use of its host plant, Psidium cattleianum, which is endemic from Brazil and has been introduced in other countries to be used in garden landscaping (Vitorino et al. 2000).

In relation to their origin, 77 galling species are proposed as endemic in Brazil. Among them, 51 are considered endemic in the Atlantic Forest, 14 were identified in species - Dasineura copacabanensis Maia, 1993; D. gigantea Angelo \& Maia, 1999; D. globosa Maia, 1993; D. marginalis Maia, 2005; Jorgenseniella eugeniae Maia, 2005; Myrciamyia maricaensis Maia, 1995; Neomitranthella robusta Maia, 1996; Stephomyia clavata (Tavares, 1920); S. espiralis Maia, 1993; S. mina Maia, 1993; S. rotundifoliorum 1993; and S. tetraloba 1993 (Diptera, Cecidomyiidae); Pacholenus pelliceus Boheman, 1836 (Coleoptera, Curculionidae) and Tectococcus ovatus Hempel, 1900 (Hemiptera, Eriococcidae). Concerning the conservational status, data are not sufficient to enable an evaluation.

Besides the inducers, other dwellers have been recorded as parasitoids, inquilines and predators (Table 5). Parasitoids were recorded in only 26 morphotypes (about 24\%), all induced by Cecidomyiidae. They are represented by seven families of Hymenoptera: Eulophidae (14 gall morphotypes), Eurytomidae (7), Platygastridae (7), Eupelmidae (4), Torymidae (4), Scelionidae (1) and Braconidae (1). None parasitoid was identified in species. Five parasitoid records were presented in genus, four in subfamily, 26 in family and nine in order. The number of records is greater than the number of parasited gall morphotypes due to multiparasitism. Rileya Ashmead, 1888 (Eurytomidae), Donquickeia Marsh, 1993 (Braconidae), Chrysonotomia Ashmead, 1904 (Eulophidae) and Proacrias Ihering, 1914 (Eulophidae) were the reported genera, all found in a single gall morphotype, except the first, found in two morphotypes. Multiparasitisme have been recorded in nine gall morphotypes.
Inquilines were recorded in 17 gall morphotypes. They were represented by Diptera, Lepidoptera, Hymenoptera, Coleoptera, Hemiptera, and Thysanoptera. All morphotypes hosted a single inquilinous order, except two of them (1. bud or leaf vein gall on Myrcia splendes, and 2) marginal roll on Neomitranthes obscura). Diptera were found in five gall morphotypes, Lepidoptera and Hymenoptera in three each, Coleoptera and Hemiptera in two each, and Thysanoptera in one. Diptera were represented by Cecidomyiidae (Dasineura tavaresi Maia, 1995, Resseliella sp. and Trotteria sp.) and Sciaridae. Lepidoptera included Stenoma annosa (Butler, 1877) (Depressidae) found in two gall morphotypes on Neomitranthes obscura. Stenoma annosa is a free-living species, whose caterpillar feeds on leaves of several Myrtaceae. Eventually, it feeds on galls, causing their complete destruction, which results in the death of the galling species. Besides, other record, but in order category, is known on Psidium cattleianum. Hymenoptera included Eulophidae (Aprostocetus sp. and Tetrastichinae sp.), Formicidae (Leptothorax sp.) and a record $\mathrm{n}$ order category. Coleoptera were represented only by Curculionidae, Hemiptera included Coccidae and Membracidae, and Thysanoptera Gynaikothrips uzeli Zimmermann, 1900.

Predators were recorded in five gall morphotypes and they included Lestodiplosis sp. (Cecidomyiidae) found in four gall morphotypes, and Novohorus sp. (Olpiidae), a pseudoscorpion found in a single morphotype.

Additionally, Bregonci et al. (2010) reported Thysanoptera and Formicidae (Hymenoptera) in galls of Cecidomyiidae on Myrciaria floribunda, but their habits were not informed.

Three gall morphotypes are described for the first time on Myrciaria floribunda: lenticular, globoid and rosette galls, all found in the Parque Municipal Chico Mendes (Rio de Janeiro, RJ). New occurrences of gall midges species are provided herein: Eugeniamyia dispar in the State of Rio de Janeiro, Dasineura myrciariae and Myrciariamyia bivalva

Table 5. Parasitoids, inquilines and predators recorded in insect galls on Myrtaceae in Brazilian restingas.

\begin{tabular}{|c|c|c|c|}
\hline Dwellers habit & Host Plant & Gall morphotype & Galling insect \\
\hline \multicolumn{4}{|l|}{ Parasitoids } \\
\hline \multicolumn{4}{|l|}{ Order: } \\
\hline \multirow[t]{9}{*}{ Hymenoptera } & Eugenia astringens Cambess. & Lenticular & $\begin{array}{l}\text { Dasineura globosa Maia, } 1995 \\
\text { (Cecidomyiidae) }\end{array}$ \\
\hline & Eugenia hiemalis Cambess. & Marginal roll & $\begin{array}{l}\text { Stephomyia cfr. clavata (Tavares, 1920) } \\
\text { (Cecidomyiidae) }\end{array}$ \\
\hline & $\begin{array}{l}\text { Eugenia punicifolia (Kunt) } \\
\text { DC. }\end{array}$ & Cylindrical & Stephomyia sp. \\
\hline & & Globoid & Undetermined \\
\hline & Myrcia splendens (SW.) DC. & Conical & Undetermined \\
\hline & & Globoid & Undetermined \\
\hline & & Fusiform & Cecidomyiidae \\
\hline & $\begin{array}{l}\text { Myrciaria floribunda (West ex } \\
\text { Willdenow) Berg }\end{array}$ & Star-shaped & Cecidomyiidae \\
\hline & $\begin{array}{l}\text { Neomitranthes obscura (DC.) } \\
\text { N. J. E. Silveira }\end{array}$ & Fusiform & $\begin{array}{l}\text { Stephomyia mina Maia, } 1993 \\
\text { (Cecidomyiidae) }\end{array}$ \\
\hline
\end{tabular}


Continuation Table 1.

\begin{tabular}{|c|c|c|c|}
\hline Dwellers habit & Host Plant & Gall morphotype & Galling insect \\
\hline \multicolumn{4}{|l|}{ Family: } \\
\hline \multirow[t]{9}{*}{ Eulophidae } & Eugenia astringens & Claviform & Stephomyia clavata \\
\hline & & Marginal roll & $\begin{array}{l}\text { Dasineura marginalis Maia, } 2005 \\
\text { (Cecidomyiidae, Diptera) }\end{array}$ \\
\hline & & Spiraled & $\begin{array}{l}\text { Stephomyia espiralis Maia, } 1993 \\
\text { (Cecidomyiidae) }\end{array}$ \\
\hline & Eugenia hiemalis & Claviform & Stephomyia cfr. clavata \\
\hline & & Conical & Cecidomyiidae \\
\hline & Myrcia ovata Cambess. & Globoid & Dasineura sp. (Cecidomyiidae) \\
\hline & & Ovoid & $\begin{array}{l}\text { Myrciamyia maricaensis Maia, } 1995 \\
\text { (Cecidomyiidae) }\end{array}$ \\
\hline & Myrciaria floribunda & Marginal roll & $\begin{array}{l}\text { Dasineura myrciariae Maia, } 1995 \\
\text { (Cecidomyiidae) }\end{array}$ \\
\hline & Neomitranthes obscura & Pineapple-shaped & $\begin{array}{l}\text { Neomitranthella robusta Maia, } 1995 \\
\text { (Cecidomyiidae) }\end{array}$ \\
\hline \multirow[t]{2}{*}{ Eupelmidae } & Eugenia astringens & Cylindrical & Stephomyia rotundifoliorum \\
\hline & Eugenia hiemalis & Claviform & Stephomyia cfr. clavata \\
\hline \multirow{4}{*}{ Eurytomidae } & & Spiraled & Stephomyia espiralis \\
\hline & Eugenia uniflora & Conical & Clinodiplosis profusa \\
\hline & & Globoid & $\begin{array}{l}\text { Eugeniamyia dispar Maia, Mendonça \& } \\
\text { Romanovski, } 1996 \text { (Cecidomyiidae) }\end{array}$ \\
\hline & Myrciaria floribunda & Globoid & Cecidomyiidae \\
\hline \multirow[t]{2}{*}{ Platygastridae } & Eugenia hiemalis & Claviform & Stephomyia cfr. clavata \\
\hline & & Cylindrical & Stephomyia sp. \\
\hline \multirow[t]{5}{*}{ Scelionidae } & Eugenia speciosa Cambess. & Claviform & Schizomyiina (Cecidomyiidae) \\
\hline & Eugenia uniflora & Conical & Clinodiplosis profusa \\
\hline & Myrcia ovata & Ovoid & Myrciamyia maricaensis \\
\hline & Myrcia splendens & Globoid & Undetermined \\
\hline & & Ovoid & Oligotrophini (Cecidomyiidae) \\
\hline \multirow[t]{4}{*}{ Torymidae } & Eugenia astringens & Cylindrical & Stephomyia rotundifoliorum \\
\hline & & Marginal roll & Dasineura marginalis \\
\hline & Eugenia copacabanensis & Rosette & Undetermined \\
\hline & Myrcia splendens & Bud & Lasiopteridi (Cecidomyiidae) \\
\hline \multicolumn{4}{|l|}{ Subfamily: } \\
\hline Tetrastichinae & Eugenia astringens & Lenticular & Dasineura globosa \\
\hline
\end{tabular}


Continuation Table 1.

\begin{tabular}{|c|c|c|c|}
\hline Dwellers habit & Host Plant & Gall morphotype & Galling insect \\
\hline \multirow[t]{2}{*}{ Tetrastichinae } & Eugenia copacabanensis & Spiraled & Stephomyia espiralis \\
\hline & Eugenia uniflora & Conical & Clinodiplosis profusa \\
\hline \multicolumn{4}{|l|}{ Genus: } \\
\hline $\begin{array}{l}\text { Chrysonotomia Ashmead, } 1904 \\
\text { (Eulophidae) }\end{array}$ & Eugenia uniflora & Conical & Clinodiplosis profusa \\
\hline Proacrias Ihering, 1914 (Eulophidae) & Myrciaria floribunda & Marginal roll & Dasineura myrciariae \\
\hline \multirow[t]{2}{*}{ Rileya Ashmead, 1888 (Eurytomidae) } & Eugenia astringens & Cylindrical & Stephomyia rotundifoliorum \\
\hline & Eugenia copacabanensis & Spiraled & Stephomyia espiralis \\
\hline \multicolumn{4}{|l|}{ Inquilines } \\
\hline Curculionidae (Coleoptera) & Neomitranthes obscura & Pineapple-shaped & Neomitranthella robusta \\
\hline Coccidae? & Myrcia splendens & Bud or leaf vein & Lasiopteridi (Cecidomyiidae) \\
\hline Membracidae (Hemiptera) & $\begin{array}{l}\text { Campomanesia guaviroba } \\
\text { (DC.) Kiaersk. }\end{array}$ & Globoid & Cecidomyiidae \\
\hline Sciaridae (Diptera) & Eugenia speciosa Cambess. & Claviform & Schizomyiina (Cecidomyiidae) \\
\hline \multicolumn{4}{|l|}{ Subfamily: } \\
\hline Tetrastichinae (Hymenoptera) & Myrcia ovata & Globoid & Dasineura sp. (Cecidomyiidae) \\
\hline \multicolumn{4}{|l|}{ Genus: } \\
\hline $\begin{array}{l}\text { Aprostocetus sp. (Eulophidae, } \\
\text { Hymenoptera) }\end{array}$ & Myrcia ovata & Ovoid & Myrciamyia maricaensis \\
\hline Trotteria sp. (Cecidomyiidae) & Myrcia splendens & Bud or leaf vein & Lasiopteridi (Cecidomyiidae) \\
\hline \multicolumn{4}{|l|}{ Species: } \\
\hline $\begin{array}{l}\text { Dasineura tavaresi Maia, } 1995 \\
\text { (Cecidomyiidae) }\end{array}$ & Neomitranthes obscura & Marginal roll & Clinodiplosis sp. (Cecidomyiidae) \\
\hline $\begin{array}{l}\text { Gynaikothrips uzeli (Zimerman, 1909) } \\
\text { (Thysanoptera, Phlaeothripidae) }\end{array}$ & Eugenia uniflora & Conical & Clinodiplosis profusa \\
\hline \multirow{2}{*}{$\begin{array}{l}\text { Stenoma annosa (Butler, 1877) } \\
\text { (Depressariidae, Lepidoptera) }\end{array}$} & Neomitranthes obscura & Marginal roll & Clinodiplosis sp. \\
\hline & Neomitranthes obscura & Pineapple-shaped & Neomitranthella robusta \\
\hline \multicolumn{4}{|l|}{ Predators: } \\
\hline \multicolumn{4}{|l|}{ Genus: } \\
\hline Lestodiplosis sp. (Cecidomyiidae) & Eugenia astringens & Marginal roll & Dasineura marginalis \\
\hline Lestodiplosis sp. & Myrcia multiflora & Marginal roll & Thysanoptera \\
\hline Lestodiplosis sp. & Myrcia splendens & Bud gall & Undetermined \\
\hline \multirow[t]{2}{*}{ Lestodiplosis sp. } & Myrciaria floribunda & Marginal roll & Dasineura myrciariae \\
\hline & Myrcia multiflora (Lam.) DC. & Marginal roll & Thysanoptera \\
\hline \multicolumn{4}{|l|}{ Species: } \\
\hline $\begin{array}{l}\text { Novohorus sp. (Olpiidae, } \\
\text { Pseudoscorpiones) }\end{array}$ & Eugenia astringens & Cylindrical & Stephomyia rotundifoliorum \\
\hline
\end{tabular}


in the municipality of Rio de Janeiro, and Neolasioptera eugeniae in the Parque Municipal Chico Mendes.

Furthermore, the globoid gall of stems of Eugenia uniflora is reported for the first time in the State of Rio de Janeiro (it was previously known only in Espírito Santo and the galler is unknown). And finally, the records of star-shaped galls (induced by Cecidomyiidae) and leaf rolls (induced by Thysanoptera) on Myrciaria floribunda are new in the municipality of Rio de Janeiro.

The morphological characterization of galls on Myrtaceae in Brazilian restingas is compiled and presented below in alphabetical order of the host plant species. Data on galled organ, gall shape, color, indumentum, number of internal chamber, galler and other dwellers are presented whenever available. Informations about their geographical distribution in Brazilian restingas are added.

Blepharocalyx salicifolius (Kunth) O. Berg.

1. Gall on leaf vein, woody, fusiform. Galler: undetermined. Locality: SP (Bertioga). Reference: Maia et al. (2008).

Calyptranthes brasiliensis Spreng.

1. Gall on bud, conical, green or brown, glabrous, multichambered. Galler: undetermined. Locality: RJ (Mangaratiba: Ilha da Marambaia). Reference: Rodrigues et al. (2014).

2. Gall on stem. Galler: undetermined. Locality: RJ (Parque Nacional da Restinga de Jurubatiba). Reference: Monteiro et al. (2004).

Campomanesia guaviroba (DC.) Kiaersk.

1. Gall on leaf vein, globoid, one-chambered. Galler: Clinodiplosis sp. (Cecidomyiidae). Locality: SP (Bertioga). Other dwellers: Membracidae (Hemiptera) - inquiline. Reference: Maia et al. (2008).

Eugenia astringens Cambess.

1. Gall on leaf, marginal roll, green, glabrous, one-chambered. Galler: Dasineura marginalis Maia, 2005 (Cecidomyiidae, Diptera). Other dwellers: Lestodiplosis sp. (Cecidomyiidae) - predator; Tetrastichinae (Eulophidae), Torymidae (Hymenoptera) - parasitoids. Localities: RJ (Mangaratiba: Ilha da Marambaia; Rio de Janeiro: Grumari; Maricá, Saquarema, Araruama, Cabo Frio, Arraial do Cabo, Carapebus, Parque Nacional da Restinga de Jurubatiba. References: Maia (2001a), Maia et al. (2005); Monteiro et al. (1994), Monteiro et al. (2004), Oliveira \& Maia (2005), Carvalho-Fernandes et al. (2016), Silva \& Maia (2016).

2. Gall on bud, cylindrical, brown, glabrous, one-chambered. Galler: Stephomyia rotundifoliorum Maia, 1993 (Cecidomyiidae). Other dwellers: Rileya sp. (Eurytomidae), Donquickeia (Braconidae), Eupelmidae, Eulophidae, Scelionidae, Torymidae (Hymenoptera) - parasitoids; Novohorus sp. (Pseudoscorpiones: Olpiidae) - predators. Localities: RJ (Maricá, Saquarema, Araruama, Cabo Frio, Arraial do Cabo, Carapebus, Parque Nacional da Restinga de Jurubatiba, São João da Barra). References: Maia (1993b, 2001a), Monteiro et al. (1994), Carvalho-Fernandes et al. (2016).

3. Gall on leaf, lenticular, yellow, glabrous, one-chambered. Galler: Dasineura globosa Maia, 1995 (Cecidomyiidae). Other dwellers: Resseliella sp. (Cecidomyiidae) - inquiline; Hymenoptera - parasitoids. Localities: RJ (Mangaratiba: Ilha da Marambaia; Rio de Janeiro: Grumari; Maricá, Saquarema, Carapebus, Araruama, Cabo Frio, Arraial do Cabo, Parque Nacional da Restinga de Jurubatiba, São João da Barra). References: Maia (1995, 2001a), Monteiro et al. (2004); Oliveira \& Maia (2005); Rodrigues et al. (2014), CarvalhoFernandes et al. (2016), Silva \& Maia (2016).

4. Gall on leaf, conical. Galler: Cecidomyiidae. Locality: RJ (Parque Nacional da Restinga de Jurubatiba). Reference: Monteiro et al. (2004).

5. Gall on leaf, conical. Galler: Cecidomyiidae. Locality: RJ (Parque Nacional da Restinga de Jurubatiba). Reference: Monteiro et al. (2004).

6. Gall on leaf, claviform, reddish, glabrous, one-chambered. Galler: Stephomyia clavata (Tavares 1920) (Cecidomyiidae). Other dwellers: Eulophidae, Eurytomidae (Hymenoptera) parasitoids. Localities: RJ (Rio de Janeiro, Carapebus, Parque Nacional da Restinga de Jurubatiba, Araruama, Arraial do Cabo, São João da Barra. References: Maia (2001a), Monteiro et al. (2004); Carvalho-Fernandes et al. (2016), Silva \& Maia (2016).

7. Gall on stem, fusiform. Galler: undetermined. Locality: RJ (Parque Nacional da Restinga de Jurubatiba). Reference: Monteiro et al. (2004).

8. Gall on leaf, globoid, green, glabrous, one-chambered. Galler: undetermined. Localities: RJ (Araruama, Arraial do Cabo). Reference: Carvalho-Fernandes et al. (2016).

9. Gall on leaf, lenticular, green, glabrous, one-chambered. Galler: Jorgenseniella eugeniae Maia, 2005 (Cecidomyiidae). Other dwellers: Trotteria sp. (Cecidomyiidae) - inquilines. Localities: SP (Bertioga), RJ (Cabo Frio, Macaé). References: Maia et al. $(2005,2008)$.

Eugenia copacabanensis Kiaersk.

1. Gall on leaf, spiraled, reddish, glabrous, one-chambered. Galler: Stephomyia espiralis Maia, 1993 (Cecidomyiidae). Other dwellers: Rileya sp. (Eurytomidae), Tetrastichinae (Eulophidae) (Hymenoptera) - parasitoids. Localities: RJ (Mangaratiba: Ilha da Marambaia; Maricá, Araruama, Cabo Frio, Arraial do Cabo. References: Maia (1993b), Maia (2001a), Monteiro et al. (2004), Carvalho-Fernandes et al. (2016).

2. Gall on bud, conical, green, glabrous, pedunculated. Galler: Dasineura copacabanensis Maia, 1993 (Cecidomyiidae). Localities: RJ (Maricá, Saquarema, Araruama, Cabo Frio, Arraial do Cabo, São João da Barra). References: Maia (1993a), Monteiro et al. (2004), Carvalho-Fernandes et al. (2016).

3. Gall on young leaf, leaf roll, reddish, glabrous, one-chambered. Galler: Dasineura sp. (Cecidomyiidae). Locality: RJ (Maricá). Reference: Maia et al. (2002).

4. Gall on leaf. Galler: Cecidomyiidae. Locality: RJ (Maricá). Reference: Monteiro et al. (1994).

5. Gall on stem. Galler: Cecidomyiidae. Locality: RJ (Maricá). Reference: Monteiro et al. (1994).

6. Gall on stem. Galler: Hymenoptera. Locality: RJ (Arraial do Cabo). Reference: Monteiro et al. (1994). 
7. Gall on leaf, fusiform, reddish, glabrous, one-chambered. Galler: Stephomyia tetraloba Maia, 1993 (Cecidomyiidae). Localities: RJ (Maricá, Arraial do Cabo). References: Maia (2001a), Carvalho-Fernandes et al. (2016).

8. Gall on leaf, conical, reddish, glabrous, one-chambered. Galler: Bruggmannia sp. (Cecidomyiidae). Locality: RJ (Arraial do Cabo). Other dwellers: Eulophidae, Eurytomidae (Hymenoptera) - parasitoids. Reference: Carvalho-Fernandes et al. (2016).

9. Gall on leaf, lenticular, green, glabrous, one-chambered. Galler: undetermined. Locality: RJ (Mangaratiba: Ilha da Marambaia). Reference: Carvalho-Fernandes et al. (2016).

10. Gall on leaf, marginal roll, green, glabrous, one-chambered. Galler: undetermined. Localities: RJ (Araruama, Cabo Frio, Arraial do Cabo). Reference: Carvalho-Fernandes et al. (2016).

11. Gall on leaf vein, globoid, green, glabrous, one-chambered. Galler: Hymenoptera. Locality: RJ (Arraial do Cabo). Reference: Carvalho-Fernandes et al. (2016).

12. Gall on bud, rosette, green, glabrous, one-chambered. Galler: undetermined. Other dwellers: Torymidae (Hymenoptera) - parasitoids. Locality: RJ (Arraial do Cabo). Reference: Carvalho-Fernandes et al. (2016).

Eugenia hiemalis Cambess.

1. Gall on bud, cylindrical, brown, glabrous, one-chambered. Galler: Stephomyia sp. (Cecidomyiidae). Other dwellers: Leptothorax sp. (Formicidae, Hymenoptera) - inquiline; Eupelmidae, Platygastridae (Hymenoptera) - parasitoids. Locality: RJ (Carapebus). Reference: Maia (2001a).

2. Gall on leaf, lenticular, green, glabrous, one-chambered. Galler: Lasiopteridi (Cecidomyiidae). Other dwellers: Hymenoptera - parasitoids. Locality: RJ (Carapebus). Reference: Maia (2001a).

3. Gall on leaf, marginal roll, green, glabrous, one-chambered. Galler: Cecidomyiidae. Other dwellers: Eulophidae (Hymenoptera) - parasitoids. Locality: RJ (Carapebus). Reference: Maia (2001a).

4. Gall on leaf, conical, yellow, glabrous, one-chambered. Galler: Cecidomyiidae. Other dwellers: Eupelmidae, Eulophidae (Hymenoptera) - parasitoids. Locality: RJ (Carapebus). Reference: Maia (2001a).

5. Gall on leaf, claviform, green or reddish, glabrous, onechambered. Galler: Stephomyia cfr. clavata (Tavares, 1920) (Cecidomyiidae). Other dwellers: Eulophidae, Eupelmidae, Platygastridae (Hymenoptera) - parasitoids. Locality: RJ (Carapebus). Reference: Maia (2001a).

6. Gall on leaf, conical, yellow, glabrous, one-chambered. Galler: Cecidomyiidae. Locality: RJ (Carapebus). Reference: Maia (2001a).

\section{Eugenia monosperma Vell.}

1. Gall on leaf, globoid, yellow, glabrous, one-chambered. Galler: undetermined. Locality: SP (Bertioga). Reference: Maia et al. (2008).

2. Gall on leaf, lenticular, glabrous, one-chambered. Galler: undetermined. Locality: SP (Bertioga). Reference: Maia et al. (2008).
Eugenia punicifolia (Kunt) DC.

1. Gall on leaf, cylindrical, green or reddish, glabrous, onechambered. Galler: Stephomyia sp. (Cecidomyiidae). Other dwellers: Hymenoptera - parasitoids. Locality: RJ (Mangaratiba: Ilha da Marambaia). Reference: Rodrigues et al. (2014).

2. Gall on leaf, lenticular, green, glabrous, one-chambered. Galler: Cecidomyiidae. Locality: RJ (Mangaratiba: Ilha da Marambaia). Reference: Rodrigues et al. (2014).

3. Gall on leaf. Galler: Cecidomyiidae. Locality: RJ (Arraial do Cabo). Reference: Monteiro et al. (1994).

4. Gall on stem, fusiform, brown, glabrous, multichambered. Galler: Lasiopteridi (Diptera, Cecidomyiidae). Other dwellers: Hymenoptera - parasitoids. Localities: RJ (Mangaratiba: Ilha da Marambaia; Rio de Janeiro: Grumari; Saquarema, Cabo Frio). References: Oliveira \& Maia (2005), Rodrigues et al. (2014), Carvalho-Fernandes et al. (2016).

5. Gall on stem. Galler: Hymenoptera. Locality: RJ (Maricá). Reference: Monteiro et al. (1994).

6. Gall on stem. Galler: undetermined. Locality: RJ (Parque Nacional da Restinga de Jurubatiba). Reference: Monteiro et al. (2004).

7. Gall on fruit, globoid, green or yellow, glabrous, multichambered. Galler: undetermined. Other dwellers: Curculionidae (Coleoptera) - inquiline; Hymenoptera parasitoids. Locality: RJ (Mangaratiba: Ilha da Marambaia). Reference: Rodrigues et al. (2014).

8. Gall on fruit. Galler: Cecidomyiidae. Locality: RJ (Maricá). Reference: Monteiro et al. (1994).

Eugenia selloi (O. Berg.) B. D. Jacks.

1. Gall on leaf, marginal roll, green, glabrous, one-chambered. Galler: Cecidomyiidae. Localities: RJ (Saquarema, Araruama, Arraial do Cabo). Reference: Carvalho-Fernandes et al. (2016).

Eugenia speciosa Cambess.

1. Gall on leaf, conical, yellow, glabrous, one-chambered. Galler: Schizomyiina (Cecidomyiidae). Other insects: Sciaridae inquiline. Locality: SP (Bertioga). Reference: Maia et al. (2008).

2. Gall on leaf, claviform, green, glabrous, one-chambered. Galler: Schizomyiina (Cecidomyiidae). Other dwellers: Platygastridae (Hymenoptera) - parasitoids. Locality: SP (Bertioga). Reference: Maia et al. (2008).

Eugenia sulcata Spring ex Mart.

1. Gall on bud, cylindrical, reddish, glabrous, one-chambered. Galler: undetermined. Locality: SP (Bertioga). Reference: Maia et al. (2008)

2. Gall on bud, ovoid, green, glabrous. Galler: undetermined. Locality: SP (Bertioga). Reference: Maia et al. (2008).

Eugenia uniflora $\mathrm{L}$.

1. Gall on leaf, globoid, spongy, whitish, glabrous, onechambered. Galler: Eugeniamyia dispar Maia, Mendonça \& Romanovski, 1996 (Cecidomyiidae). Other dwellers: Eurytomidae (Hymenoptera) - parasitoids. Localities: RJ (Parque Natural Municipal Chico Mendes - new record), SP (Bertioga). Reference: Maia et al. (2008). 
2. Gall on leaf, conical, green or reddish, glabrous, onechambered. Galler: Clinodiplosis profusa Maia 2001 (Cecidomyiidae). Other dwellers: Chrysonotomyia sp. (Eulophidae), Tetrastichinae (Eulophidae), Platygastridae (Hymenoptera) - parasitoids; Gynaikothrips uzeli (Zimerman, 1909) (Thysanoptera, Phlaeothripidae) - inquilines. Localities: RJ (Mangaratiba: Ilha da Marambaia; Rio de Janeiro: Grumari; Maricá, Saquarema, Araruama, Cabo Frio, Arraial do Cabo, Carapebus, Parque Nacional da Restinga de Jurubatiba, São João da Barra). References: Maia (2001a,b); Monteiro et al. (1994), Monteiro et al. (2004), Oliveira \& Maia (2005), Carvalho-Fernandes et al. (2016), Silva \& Maia (2016).

3. Gall on leaf, lenticular, green, yellowish or black, glabrous, one-chambered. Galler: Neolasioptera eugeniae Maia 1993 (Cecidomyiidae). Other dwellers: Eulophidae (Cecidomyiidae) - parasitoids. Localities: RJ (Paraty, Angra dos Reis: Ilha Grande; Rio de Janeiro: Grumari, Parque Natural Municipal Chico Mendes - new record; Maricá, Saquarema, Araruama, Cabo Frio, Arraial do Cabo, São João da Barra. References: Maia (1993a, 2001a), Maia \& Oliveira (2010), Monteiro et al. (1994), Oliveira \& Maia (2005), Carvalho-Fernandes et al. (2016), Silva \& Maia (2016).

4. Gall on fruit, conical, reddish, glabrous, one-chambered. Galler: Cecidomyiidae. Other dwellers: Eurytomidae (Hymenoptera) - parasitoids. Localities: RJ (Maricá, Cabo Frio, São João da Barra). References: Monteiro et al. (1994), Carvalho-Fernandes et al. (2016).

5. Gall on leaf, conical, green, glabrous, one-chambered. Galler: Eugeniamyia triangularis Maia, 2011 (Cecidomyiidae). Locality: RJ (Maricá). Reference: Maia \& Nava (2011).

6. Gall on bud flower, conical, reddish, glabrous, one-chambered. Galler: Cecidomyiidae. Locality: RJ (Rio de Janeiro: Grumari). Reference: Oliveira \& Maia (2005).

7. Gall on stem, fusiform, brown, glabrous, one-chambered. Galler: undetermined. Localities: RJ (Angra dos Reis: Ilha Grande; Mangaratiba: Ilha da Marambaia). References: Maia \& Oliveira (2010), Rodrigues et al. (2014).

8. Gall on leaf, fold, green, glabrous, one-chambered. Galler: Cecidomyiidae. Locality: RJ (Mangaratiba: Ilha da Marambaia). Reference: Rodrigues et al. (2014).

Myrcia brasiliensis Kiaersk.

1. Gall on stem, fusiform, brown, glabrous, one-chambered. Galler: Pacholenus pelliceus Boheman, 1836 (Coleoptera: Curculionidae). Locality: RJ (Maricá). Reference: Monteiro et al. (1994).

2. Gall on bud, ovoid, multichambered, glabrous. Galler: undetermined. Locality: SP (Bertioga). Reference: Maia et al. (2008).

3. Gall on leaf, lenticular, green, glabrous, one-chambered. Galler: Bruggmanniella cfr. (Cecidomyiidae). Locality: SP (Bertioga). Reference: Maia et al. (2008).

Myrcia ilheosensis Kiaersk.

1. Gall on stem, fusiform, brown, glabrous, one-chambered. Galler: Pacholenus pelliceus Boheman, 1836 (Coleoptera: Curculionidae). Localities: SP (Bertioga), RJ (Maricá).
References: Monteiro et al. (1994), Vanin (2008), Maia et al. (2008).

Myrcia lundiana Kiaersk.

1. Gall on leaf, globoid. Galler: Dasineura sp. (Cecidomyiidae). Locality: RJ (Parque Nacional da Restinga de Jurubatiba). Reference: Monteiro et al. (2004).

2. Gall on leaf. Galler: undetermined. Locality: RJ (Maricá). Reference: Monteiro et al. (1994).

3. Gall on bud, ovoid, grooved. Galler: Myrciamyia maricaensis Maia, 1995 (Cecidomyiidae). Locality: RJ (Parque Nacional da Restinga de Jurubatiba). References: Maia (1996), Monteiro et al. (2004).

4. Gall on peduncle flower, fusiform. Galler: Cecidomyiidae. Locality: RJ (Parque Nacional da Restinga de Jurubatiba). Reference: Monteiro et al. (2004).

5. Gall on leaf, lenticular. Galler: undetermined. Locality: RJ (Parque Nacional da Restinga de Jurubatiba). Reference: Monteiro et al. (2004).

6. Gall on leaf vein. Galler: undetermined. Locality: RJ (Parque Nacional da Restinga de Jurubatiba. Reference: Monteiro et al. (2004).

Myrcia multiflora (Lam.) DC.

1. Gall on leaf, marginal roll, green, glabrous, one-chambered. Galler: Thysanoptera. Other insects associados: Lestodiplosis sp. - predator: Locality: SP (Bertioga). References: Maia et al. (2008).

Myrcia ovata Camb.

1. Gall on leaf, globoid, yellow, glabrous, one-chambered. Galler: Dasineura sp. (Cecidomyiidae). Other dwellers: Tetrastichinae (Eulophidae, Hymenoptera) - inquiline. Localities: RJ (Maricá, Arraial do Cabo). References: Maia (2001a), Maia et al. (2002), Monteiro et al. (2004), Carvalho-Fernandes et al. (2016).

2. Gall on leaf. Galler: undetermined. Locality: RJ (Maricá). Reference: Monteiro et al. (1994).

3. Gall on bud, ovoid, green, grooved, glabrous, one-chambered. Galler: Myrciamyia maricaensis Maia, 1995 (Cecidomyiidae). Other dwellers: Platygastridae (Hymenoptera) - parasitoids; Aprostocetus sp. (Eulophidae, Hymenoptera) - inquiline. Localities: RJ (Maricá, Cabo Frio, Arraial do Cabo, Carapebus, Parque Nacional da Restinga de Jurubatiba). References: Maia (1996, 2001a), Monteiro et al. (2004).

4. Gall on peduncle flower, fusiform, green, glabrous, onechambered. Galler: Cecidomyiidae. Locality: RJ (Maricá). References: Maia (2001a), Monteiro et al. (2004).

5. Gall on leaf vein, conical, green, glabrous, one-chambered. Galler: Dasineura sp. (Cecidomyiidae). Localities: RJ (Araruama, Arraial do Cabo). Reference: Carvalho-Fernandes et al. (2016).

6. Gall on leaf, conical, green, glabrous, one-chambered. Galler: Cecidomyiidae. Locality: RJ (Arraial do Cabo). Reference: Carvalho-Fernandes et al. (2016).

7. Gall on leaf, marginal roll, green, glabrous, one-chambered. Galler: Thysanoptera. Locality: RJ (Cabo Frio). Reference: Carvalho-Fernandes et al. (2016). 
8. Gall on leaf, lenticular, green, glabrous, one-chambered. Galler: undetermined. Locality: RJ (Cabo Frio). Reference: Carvalho-Fernandes et al. (2016).

Myrcia palustris DC.

1. Gall on leaf, lenticular, green, glabrous, one-chambered. Galler: undetermined. Locality: SP (Bertioga). Reference: Maia et al. (2008).

2. Gall on bud, ovoid, brown, glabrous, multichambered. Galler: undetermined. Locality: SP (Bertioga). Reference: Maia et al. (2008).

Myrcia racemosa (O. Berg.) Kiaersk.

1. Gall on stem, globoid, brown, glabrous, multichambered. Galler: undetermined. Locality: RJ (Mangaratiba: Ilha da Marambaia). Reference: Rodrigues et al. (2014).

Myrcia splendens (SW.) DC.

1. Gall on leaf, globoid, green, glabrous, one-chambered. Galler: undetermined. Other dwellers: Hymenoptera - parasitoids. Locality: RJ (Mangaratiba: Ilha da Marambaia). Reference: Rodrigues et al. (2014).

2. Gall on leaf, conical, yellow or green, glabrous, one-chambered. Galler: undetermined. Other dwellers: Hymenoptera parasitoids. Locality: RJ (Mangaratiba: Ilha da Marambaia). Reference: Rodrigues et al. (2014).

3. Gall on leaf, marginal roll, green, glabrous, one-chambered. Galler: undetermined. Locality: RJ (Mangaratiba: Ilha da Marambaia). Reference: Rodrigues et al. (2014).

4. Gall on leaf, leaf roll, reddish, glabrous, one-chambered. Galler: undetermined. Locality: SP (Bertioga). Reference: Maia et al. (2008).

5. Gall on bud, globoid, green or brown, glabrous, multichambered. Galler: undetermined. Locality: RJ (Mangaratiba: Ilha da Marambaia). Reference: Rodrigues et al. (2014).

6. Gall on stem, globoid, brown, glabrous. Galler: Lasiopteridi (Cecidomyiidae). Other dwellers: Platygastridae (Hymenoptera) - parasitoids. Locality: SP (Bertioga). Reference: Maia et al. (2008).

7. Gall on vein leaf, globoid, brown, glabrous, one-chambered. Galler: Lasiopteridi (Cecidomyiidae). Other dwellers: Platygastridae (Hymenoptera) - parasitoids. Locality: SP (Bertioga). Reference: Maia et al. (2008).

8. Gall on bud. Galler: Lasiopteridi (Cecidomyiidae). Other dwellers: Torymidae (Hymenoptera) - parasitoids. Locality: SP (Bertioga). Reference: Maia et al. (2008).

9. Gall on stem, fusiform, multichambered. Galler: Cecidomyiidae. Locality: SP (Bertioga). Reference: Maia et al. (2008).

10. Gall on stem or leaf vein. Galler: Lasiopteridi (Cecidomyiidae). Other dwellers: Hemiptera (Coccidae?), Trotteria sp. (Cecidomyiidae) - inquilines. Locality: SP (Bertioga). Reference: Maia et al. (2008).

11. Gall on bud, conical. Galler: undetermined. Other dwellers: Lestodiplosis sp.(Cecidomyiidae) - predator. Locality: SP (Bertioga). Reference: Maia et al. (2008).

\section{Myrciaria floribunda Miq}

1. Gall on leaf, marginal roll, green, glabrous, one-chambered. Galler: Dasineura myrciariae Maia, 1995 (Cecidomyiidae). Other dwellers: Lestodiplosis sp. (Cecidomyiidae) - predator;
Proacrias sp. (Eulophidae, Hymenoptera) - parasitoids. Localities: RJ (Rio de Janeiro: Parque Natural Municipal Chico Mendes - new record; Maricá, Carapebus, Parque Nacional da Restinga de Jurubatiba), ES (Guarapari). References: Maia (1995), Monteiro et al. (1994), Monteiro et al. (2004), Bregonci et al. (2010).

2. Gall on leaf, leaf roll, green, glabrous, one-chambered. Galler: Thysanoptera. Localities: RJ (Rio de Janeiro: Parque Natural Municipal Chico Mendes - new record; Maricá, Arraial do Cabo). References: Monteiro et al. (1994), Carvalho-Fernandes et al. (2016).

3. Gall on bud, bivalve, yellow, glabrous, one-chambered. Galler: Myrciariamyia bivalva Maia, 1994 (Cecidomyiidae). Localities: RJ (Rio de Janeiro: Parque Natural Municipal Chico - new record; Maricá, Carapebus, Arraial do Cabo, Jurubatiba). References: Maia (1994, 2001a), Monteiro et al. (1994), Monteiro et al. (2004).

4. Gall on leaf, star-shaped, yellow, one-chambered. Galler: Cecidomyiidae. Other dwellers: Hymenoptera - parasitoids. Localities: RJ (Rio de Janeiro: Parque Natural Municipal Chico Mendes - new record; Maricá, Carapebus). Reference: Maia (2001a).

5. Gall on leaf, lenticular, green, glabrous, one-chambered. Galler: undetermined. Locality: RJ (Rio de Janeiro: Parque Natural Municipal Chico Mendes - new record).

6. Gall on leaf, globoid, glabrous, one-chambered. Galler: Eugeniamyia dispar Maia, Mendonça \& Romanovski, 1996 (Cecidomyiidae). Locality: (Rio de Janeiro: Parque Natural Municipal Chico Mendes - new record).

7. Gall on stem, globoid, green, glabrous, one-chambered. Galler: undetermined. Localities: RJ (Rio de Janeiro: Parque Natural Municipal Chico Mendes - new record), ES (Guarapari). Reference: Bregonci et al. (2010).

8. Gall on leaf, globoid, green, hairy, one-, bi- or three-chambered. Galler: Cecidomyiidae. Other dwellers: Prodecatoma (Eurytomidae, Hymenoptera) - parasitoids, Thysanoptera and Formicidae (no data on habits). Locality: ES (Guarapari). Reference: Bregonci et al. (2010).

9. Gall on leaf, cylindrical, green, glabrous, one-chambered Galler: Cecidomyiidae. Locality: RJ (Arraial do Cabo). Reference: Carvalho-Fernandes et al. (2016).

10. Gall on bud, rosette, green, glabrous, one-chambered. Galler: undetermined. Locality: RJ (Parque Natural Municipal Chico Mendes - new record).

Myrciaria tenella (DC.) O. Berg

1. Gall on young leaf, leaf fold, green, glabrous, one-chambered. Galler: Hemiptera. Localities: RJ (Araruama, São João da Barra). Reference: Carvalho-Fernandes et al. (2016).

Neomitranthes obscura (DC.) Silveira

1. Gall on leaf, conical, green, glabrous, one-chambered. Galler: Cecidomyiidae. Localities: RJ (Maricá, Arraial do Cabo, Carapebus e Parque Nacional da Restinga de Jurubatiba). References: Maia \& Couri (1997), Maia (2001a), Monteiro et al. (2004).

2. Gall on leaf, leaf roll, green, glabrous, one-chambered Galler: Thysanoptera. Localities: RJ (Maricá, Araruama, Arraial 
do Cabo). References: Maia 2006, Monteiro et al. (1994), Carvalho-Fernandes et al. (2016).

3. Gall on leaf, fusiform, green, glabrous, one-chambered. Galler: Stephomyia mina Maia, 1993 (Cecidomyiidae). Other dwellers: Hymenoptera - parasitoids. Localities: RJ (Maricá, Araruama, Arraial do Cabo, Carapebus). References: Maia (1993b, 2001a), Monteiro et al. (2004), Carvalho-Fernandes et al. (2016).

4. Gall on leaf, roll marginal, green, glabrous, one-chambered. Galler: Clinodiplosis sp. (Cecidomyiidae). Other dwellers: Dasineura tavaresi Maia, 1995 (Cecidomyiidae), Stenoma annosa Butler, 1877 (Lepidoptera) - inquilines. Localities: RJ (Maricá, Saquarema, Araruama, Cabo Frio, Arraial do Cabo, Carapebus, Parque Nacional da Restinga de Jurubatiba), ES (Guarapari). References: Maia (1995, 1996, 2001a), Monteiro et al. (2004), Carvalho-Fernandes et al. (2016), Silva \& Maia (2016).

5. Gall on bud, pineapple-shaped, green, glabrous. Galler: Neomitranthella robusta Maia, 1995 (Cecidomyiidae). Other dwellers: inquilines - Stenoma annosa (Lepidoptera), parasitoids - Tetrastichinae (Eulophidae, Hymenoptera). Localities: RJ (Maricá, Saquarema, Araruama, Cabo Frio, Arraial do Cabo, Carapebus, Parque Nacional da Restinga de Jurubatiba). References: Maia (1996, 2001a), Monteiro et al. (2004), Carvalho-Fernandes et al. (2016).

6. Gall on stem, fusiform, brown, glabrous, one-chambered. Galler: undetermined. Localities: RJ (Saquarema, Araruama, Arraial do Cabo, Parque Nacional da Restinga de Jurubatiba. References: Monteiro et al. (2004), Carvalho-Fernandes et al. (2016).

\section{Plinia cauliflora Kausel}

1. Gall on leaf, marginal roll, green, glabrous, one-chambered. Galler: Cecidomyiidae. Locality: RJ (Angra dos Reis: Ilha Grande). Reference: Maia \& Oliveira (2010).

2. Gall on bud, cylindrical, green, glabrous, one-chambered. Galler: Cecidomyiidae. Locality: RJ (Angra dos Reis: Ilha Grande). Reference: Maia \& Oliveira (2010).

Psidium cattleianum Sabine

1. Gall on leaf, biconical, green, glabrous, one-chambered. Galler: Tectococcus ovatus Hempel (Eriococcidae, Hemiptera). Locality: RJ (Angra dos Reis: Ilha Grande). Reference: Maia \& Oliveira (2010).

2. Gall on leaf or bud, globoid, yellow, glabrous, one-chambered. Galler: Cecidomyiidae. Locality: RJ (Angra dos Reis: Ilha Grande). Reference: Maia \& Oliveira (2010).

3. Gall on stem, fusiform, brown, glabrous, multichambered. Galler: undetermined. Locality: RJ (Angra dos Reis: Ilha da Marambaia). Reference: Rodrigues et al. (2014).

4. Gall on leaf, tubular, green, glabrous, one-chambered. Galler: Lasiopteridi (Cecidomyiidae). Locality: SP (Bertioga). Reference: Maia et al. (2008).

5. Gall on bud, green, rosette, glabrous. Galler: Dasineura gigantea Angelo \& Maia, 1999 (Cecidomyiidae). Locality: SP (Bertioga). Reference: Maia et al. 2008.
6. Gall on leaf, conical, with small apical projections, green, glabrous. Galler: Cecidomyiidae. Locality: SP (Bertioga). Reference: Maia et al. (2008).

7. Gall on leaf, globoid, one-chambered. Galler: undetermined. Locality: SP (Bertioga). Other dwellers: Lepidoptera inquiline. Reference: Maia et al. (2008).

8. Gall on leaf or leaf vein, lenticular. Galler: Cecidomyiidae. Locality: SP (Bertioga). Reference: Maia et al. 2008.

\section{Discussion}

In Brazilian restingas, Myrtaceae included from $18.18 \%$ to $11.38 \%$ of the total number of galled plant species (Table 1). In other physiognomies of the Atlantic Forest, the rates were lower, $0.00 \%$ in Semidecidual Seasonal Forest (Flor et al. 2018) and Altitude Fields (Coelho et al. 2013), from 3.86\% to $11.11 \%$ in Ombrophilous Forest (Maia 2014, Maia et al. 2014 and Maia \& Mascarenhas 2017), 8.16\% in High Altitude Wetland Forest (Santos et al. 2011), and $9.75 \%$ in Tableland Forest (Maia \& Carvalho-Fernandes 2015). Furthermore, Myrtaceae were not the plant family with the greatest number of galled species in these physiognomies. This position is occupied by Sapindaceae (in Semidecidual Seasonal Forest - Flor et al. 2018), Asteraceae (in Altitude Fields - Coelho et al. 2013 and Ombrophilous Forest-Maia et al. 2014 and Maia \& Mascarenhas 2017), Melastomataceae (Ombrophilous Forest - Maia 2014), Nyctaginaceae (High Altitude Wetland Forest - Santos et al. 2011), and Fabaceae (in Tableland Forest - Maia \& Carvalho-Fernandes 2015). The rates of gall morphotypes on Myrtaceae varied from $34.72 \%$ to $13.13 \%$ of the total number of gall morphotypes in Brazilian restingas (Table 1). In other physiognomies of the Atlantic Forest, these rates were lower, $0.00 \%$ in Semidecidual Seasonal Forest (Flor et al. 2018) and Altitude Fields (Coelho et al. 2013), from 6.40\% to $10.89 \%$ in Ombrophilous Forest (Maia 2014, Maia et al. 2014 and Maia \& Mascarenhas 2017), $8.86 \%$ in High Altitude Wetland Forest (Santos et al. 2011), and 9.09\% in Tableland Forest (Maia \& Carvalho-Fernandes 2015). These data revealed that Myrtaceae are an important host plant family mainly in restingas, while in other physiognomies of the Atlantic Forest this family can contribute to the gall richness as in the Ombrophilous Forest, High Altitude Wetland Forest and Tableland Forest or not as in Semidecidual Seasonal Forest and Altitude Fields. Eugenia and Myrcia highlighted as the Myrtaceae genera with the greatest number of galled species and gall morphotypes. Both are important components of the restinga flora, being the best represented genera of Myrtaceae in this physiognomy (Lourenço \& Barbosa 2012, Souza \& Morim 2008). The hypothesis of taxon size (Fernandes 1992) could explain the greater insect galls richness on Myrtaceae, Eugenia and Myrcia. It predicts that richer taxa have potentially more hosts and, consequently, a greater number of associated galling insects.

Leaves have been reported as the most galled plant organ in all regions of the world. This pattern was observed for the first time by Felt 1940 and has been confirmed in several studies (Maia \& Fernandes 2004, Santos et al. 2011, Maia \& Carvalho-Fernandes 2016, Maia \& Mascarenhas 2017), probably because leaves represent an abundant, frequent and predictable resource. 
Green galls predominated, probably because this is the color of the most frequent galled organ, leaf. Most galls were glabrous and onechambered. These features have been observed as the most frequent in several Brazilian inventories, not only in restingas, but also in other physiognomies of the Atlantic Forest, as well as in other domains phytogeographic (Maia \& Fernandes 2004, Santos et al. 2011, Maia \& Carvalho-Fernandes 2016, Maia \& Mascarenhas 2017).

Only $19 \%$ of the galling species have been identified in species. These results show how the taxonomical knowledge of the gallers is still incipient. Cecidomyiidae induced most galls as in all other inventories in the world. Among the gall midges genera, Clinodiplosis Kieffer, 1894 and Dasineura Rondani, 1840 have a wide world distribution, while Neolasioptera Felt, 1908 occurs in the Nearctic and Neotropical regions, and the others, Bruggmannia Tavares, 1906, Eugeniamyia Maia, Mendonça \& Romanovski, 1997, Jorgenseniella Maia, 2005, Myrciamyia Maia, 1995, Myrciariamyia Maia, 1994, Neomitranthella Maia, 1996, and Stephomyia Rondani, 1840 are exclusively Neotropical. Among them, five have been recorded only in Brazil: Eugeniamyia, Myrciariamyia, Jorgenseniella, Myrciamyia, and Neomitranthella, being the last three restricted to restingas. Clinodiplosis, Dasineura, and Neolasioptera are speciose genera, with 476, 103 and 134 described species, respectively. Bruggmannia, Eugeniamyia, Myrciariamyia, and Stephomyia include 19, 2, 3 and 7 species, respectively. The others are monotypic (Gagné \& Jaschhof 2017). Clinodiplosis, Dasineura, and Neolasioptera induce galls on several plant families, whereas most Bruggmannia species occurs on Nyctaginaceae, the other genera are exclusively associated with Myrtaceae (Gagné \& Jaschhof 2017).

Rio de Janeiro is the most investigated Brazilian State, for this reason, it harbors most records. The geographical distribution of all galled plants is wider than that of the gallers, indicating that these insects can probably have a greater area of occurrence.

Galling species that occur exclusively on endemic plants were proposed as endemic too, based on their specificity of hosts. Concerning the conservational status, the gallers could not be evaluated as data on the host plants are deficient.

The presence of parasitoids, inquilines and predators has been frequently reported in gall inventories, not only in restingas and in Myrtaceae, but also in other ecosystems and plant families (Maia 2001a, Maia \& Fernandes 2004, Maia et al. 2014, Maia \& CarvalhoFernandes 2016). Parasitoids have been considered the most frequent natural enemies of galling insects (Gagné 1994). Although all parasitoid families found in the present study are associated with several plant families in restinga, they are more diverse and frequent on Myrtaceae (Maia \& Azevedo 2009). Concerning the parasitoids genera, Donquickeia and Proacrias are associated exclusively with Myrtaceae in restingas, while Rileya and Chrysonotomia have been recorded in other families, too (Maia \& Azevedo 2009). Most records are in family categories, revealing that their taxonomical knowledge is still poor in Brazilian restingas. Multiparasitisme have been recorded not only in Myrtaceae, but also in other plant families (Maia \& Monteiro 1999, Maia \& Tavares 2000, Maia \& Azevedo 2009). All predaceous and most inquilinous taxa recorded on galls in Myrtaceae of restingas have been reported in other plant families too (Maia 2001a, 2002), as well as in other phytogeographic domains (Maia 2001a, Maia \& Fernandes 2004, Maia et al. 2014, Maia \& Carvalho-Fernandes 2016). But some of them, as Leptothorax sp. (Hymenoptera), Stenoma annosa (Lepidoptera), and
Gynaikothrips uzeli (Thysanoptera) are known only on Myrtaceae at restingas. These diverse associated fauna highlights the importance of the galling species as ecosystem engineers, as they provide a new niche, the gall, which can be used by several arthropods.

Although restingas are the best studied phytophysiognomy of Brazil, new records were added, as many areas have not yet been studied.

\section{Conclusions}

Myrtaceae harbor a great richness of insect galls in Brazilian restingas, 111 morphotypes, in 25 host plant species (15 endemic). Most morphotypes are induced by Cecidomyiidae. Twelve gall midge species (Diptera, Cecidomyiidae), one beetle species (Coleoptera, Curculionidae) and one scale-insect species (Hemiptera, Eriococcidae) are associated with endemic hosts. These gallers are proposed as endemic.

Eugenia L. is the plant genus with the greatest richness of host species and gall morphotypes. Leaves are the most frequent galled organ. Globoid shape, green color, absence of trichomes and a single internal chamber are the predominant gall traits. Many arthropods, mainly insects, have been reported as dwellers of galls, where they play the role of parasitoids, inquilines and predators, highlighting the importance of the gallers as ecosystem engineers. The taxonomical knowledge of the galling species and other dwellers is still deficient. Great efforts are necessary to refine it, involving field trips, rearing, and taxonomist participation.

\section{Acknowledgments}

The author would like to thank CNPq (Conselho Nacional de Desenvolvimento Científico e Tecnológico) for financial suport and Dirceu Lopes de Souza (Museu Nacional/UFRJ) for field assistance.

\section{Author Contributions}

Valéria Cid Maia: Substantial contribution in the concept and design of the study; Contribution to data collection; Contribution to data analysis and interpretation; Contribution to manuscript preparation; Contribution to critical revision, adding intelectual content.

\section{Conflicts of interest}

The author declares that she has no conflict of interest related to the publication of this manuscript.

\section{References}

ARAÚJO, D.S.D. 1992. Vegetation types of sandy coastal plains of tropical Brazil: a first approximation. In: Seeliger, U. (Ed.). Coastal Plant Communities of Latin America. San Diego: Academic Press. p. 337-347.

ARAÚJO, D.S.D. \& HENRIQUES, R.P.B. 1984. Análise Florística das Restingas do Estado do Rio de Janeiro. In Restingas: Origem, Estrutura, Processos (L.D. Lacerda, D.S.D. Araújo, R. Cerqueira \& B. Turcq, Eds.). Ceuff, Niterói, P. 159-193.

ARAÚJO, D.S.D. 2000. Análise florística e fitogeográfica das restingas do Estado do Rio de Janeiro. Tese de doutorado, Universidade Federal do Rio de Janeiro, Rio de Janeiro. 
BREGONCI, J. M., POLYCARPO, P.V. \& MAIA, V.C. 2010. Galhas de insetos do Parque Estadual Paulo César Vinha (Guarapari, ES, Brasil).Biota Neotrop 10(1):265-274. http://www.biotaneotropica.org.br/v10n1/pt/abstract?inven tory+bn01410012010

BUTLER, A. G. 1877. Descriptions of new species of Cryptolechia from the Amazons. Cistula ent. 2: 163-164, 187-193.

CARVALHO-FERNANDES, S.P. \& MAIA, V. C. 2011. Registros de galhas de insetos no município de Paraty (RJ, Brasil). X Congresso de Ecologia do Brasil (on line), SEB: 1-2.

CARVALHO-FERNANDES, S.P., ASCENDINO, S., MAIA, V. C. \& COURI, M. S. 2016. Diversity of insect galls associated with coastal shrub vegetation in Rio de Janeiro, Brazil. An Acad Bras Cienc 88(3):1407-1418.

COELHO, M. S., CARNEIRO, M. A. A., BRANCO, C., BORGES, R. A X. \& FERNANDES, G. W. 2009. Gall-Inducing Insects from Campos de Altitude, Brazil. Biota Neotrop. 2013, 13(4): 139-151.

FERNANDES, G.W. 1992. Plant size family and age effects on insular gallforming species richness. Global Ecol. Biogeog. 2: 71-74.

FELT, E.P. 1940. Plant Galls and Gall Makers. Comstock Publishing Co., Ithaca, N. Y., VIII + 364P

FLORA DO BRASIL. 2020 em construção. Jardim Botânico do Rio de Janeiro. Disponível em: <http://floradobrasil.jbrj.gov.br/>. Acesso em: 22 Set. 2016.

GAGNÉ, R. J. 1994. The Gall Midges of the Neotropical Region. Cornell University Pres, Ithaca, xi +356 p., 4 pls

GAGNÉ, R.J. \& JASCHHOF, M. 2017. A Catalog of the Cecidomyiidae (Diptera) of the World. Fourth Edition. Digital. 762 pp.

GIARETTA, A. \& PEIXOTO, A. L. 2015. Myrtaceae da restinga no norte do Espírito Santo, Brasil. Bol. Mus. Biol. Mello Leitão (N. Sér.) 37(1): 45-126.

GOVAERTS, R., SOBRAL, M., ASHTON P., BARRIE, F., HOLST, B. K., LANDRUM, L. L., MATSUMOTO, K., MAZINE, F. F., LUGHADDHA, E. N., PROENÇA, C., SOARES SILVA, L. H., WILSON, P.G. \& LUCAS, E. 2014. World checklist of Myrtaceae. Royal Botanic Gardens, Kew. http:// apps.kew.org/wcsp/ (14 August 2018).

LACERDA, L. D. DE, ARAÚJO, D.S.D. \& MACIEL, N.C. 1982. Restingas brasileiras: uma bibliografia. Rio de Janeiro, Fund. José Bonifácio, 47p.

LACERDA L. D., ARAÚJO, D. S. D. \& MACIEL, N. C. 1993. Dry coastal ecosystems of the tropical Brazilian coast. Pp. 477-493. In van der Maarel E. (ed.). Dry coastal ecosystems: Africa, America, Asia and Oceania. Elsevier, Amsterdam.

LANDRUM, L.R. \& KAWASAKI, M.L. 1997. The genera of Myrtaceae in Brazil: an illustrated synoptic treatment and identification keys. Brittonia 49: 508-536.

LOURENÇO, A. R. DE L. \& BARBOSA, M. R. DE. 2012. Myrtaceae em restingas no limite norte de distribuição da Mata Atlântica, Brasil. Rodriguésia 63(2):373-393.

MAIA, V.C. 1993a. Descrição de duas espécies novas de Cecidomyiidae (Diptera) associadas a Eugenia spp. (Myrtaceae). Rev Bras Entomol 37(4):717-721.

MAIA, V.C. 1993b. Considerações sobre Stephomyia Tavares (Diptera, Cecidomyiidae, Asphondyliidi), com descrição de quatro espécies novas associadas com Eugenia L. e Neomitranthes obscura (DC.) Legr. (Myrtaceae). Rev Bras Zool 10(3):521-530.

MAIA, V.C. 1994. Myrciariamyia bivalva, gen.n. e sp.n. (Diptera, Cecidomyiidae) associado com Myrciaria floribunda (Camb.) Legr. (Myrtaceae) no Brasil. Rev Bras Zool 11(4):635-639.

MAIA, V.C. 1995. Três espécies novas de Dasineura Rondani (Diptera, Cecidomyiidae) associadas à Myrtaceae na restinga da Barra de Maricá, Rio de Janeiro. Rev Bras Zool 12(4):1010-1008.

MAIA, V.C., 1996. Dois gêneros novos de Cecidomyiidae (Diptera) associados à Myrtaceae, na restinga da Barra de Maricá, Rio de Janeiro, Brasil. Rev Bras Zool 12(3):567-574.

MAIA, V.C. 2001a. The gall midges (Diptera, Cecidomyiidae) from three restingas of Rio de Janeiro State, Brazil. Rev Bras Zool 18(2):583-629.
MAIA, V.C. 2001b. New genera and species of gall midges (Diptera, Cecidomyiidae) from three restingas of Rio de Janeiro State, Brazil. Rev Bras Zool 18(Supl. 1):1-32.

MAIA, V. C. 2002. Description of the larva of Houardodiplosis rochae Tavares, 1925 (Diptera, Cecidomyiidae, Clinodiplosini) and new record of pseudoscorpions in galls. Rev Bras Entomol 46(1):81-82

MAIA, V. C. 2005. Catálogo dos Cecidomyiidae (Diptera) do Estado do Rio de Janeiro. Biota Neotropic 5 (2):1-15. http://www.biotaneotropica.org.br/ $\mathrm{v} 5 \mathrm{n} 2 / \mathrm{pt} / \mathrm{abstract}$ inventory+BN00805022005

MAIA, V.C. 2006. Galls of Hemiptera, Lepidoptera and Thysanoptera from Central and South Americas. Publ. Avul. Mus. Nac, 110: 1-24.

MAIA, V. C. 2013. Galhas de insetos em restingas da região sudeste do Brasil com novos registros. Biota neotropic 13 (1): 183-209. http://www. biotaneotropica.org.br/v13n1/en/abstract?inventory+bn01613012013

MAIA, V. C. 2014. Insect galls of Itamonte (Minas Gerais, Brazil): characterization and occurrence. Biota Neotrop 14: 1-17. http://www. biotaneotropica.org.br/v14n1/en/ abstract?article+bn01114012014

MAIA, V.C \& AZEVEDO, M. A. P. 2009 Micro-himenópteros associados com galhas de Cecidomyiidae (Diptera) em Restingas do estado do Rio de Janeiro. Biota Neotropic 9:1-14. http://www.biotaneotropica.org.br/v9n2/ pt/abstract?article+bn02809022009

MAIA, V. C. \& BARROS, G. P.S. 2009. Espécies de Cecidomyiidae (Diptera) registradas no Estado do Rio de Janeiro, Brasil. Arq Mus Nac 67(3-4):211220.

MAIA, V. C. \& CARVALHO-FERNANDES, S. P. 2016. Insect galls of a protected remnant of the Atlantic Forest tableland from Rio de Janeiro State (Brazil) Rev. Bras. Entomol. 60: 40-56.

MAIA, V. C. \&. COURI, M. S. 1997. Cecidomyiidae (Diptera) associated with Myrtaceae at restinga of Barra de Maricá (RJ). Rev. Bras. Biol. 57(2): 227-230.

MAIA, V. C. \& FERNANDES, G. W. 2004. Insect Galls from Serra de São José (Tiradentes, MG, Brazil). Braz. J. Biol. 64: 423-445.

MAIA V. C., CARDOSO, J. L. T. \& BRAGA, J. M. A. 2014. Insect galls from Atlantic Forest areas of Santa Teresa, Espírito Santo, Brazil: characterization and occurrence. Bol. Mus. Biol. Mello Leitão (N. Sér.) 33: 47-129.

MAIA, V. C. \& MASCARENHAS, B. 2017. Insect Galls of the Parque Nacional do Itatiaia (Southeast Region, Brazil). An. Acad. Bras. Cienc. 89(1 Suppl.): 505-575

MAIA, V. C. \& MONTEIRO, R. F. 1999. Espécies cecidógenas (Diptera, Cecidomyiidae) e parasitóides (Hymenoptera) associados a Guapira opposita (Vell.) Reitz. (Nyctaginaceae) na restinga da Barra de Maricá, Rio de Janeiro. Rev. Bras. Zool. 16(2):483-487.

MAIA, V. C. \& NAVA, D. E. 2011. New gall midges (Diptera, Cecidomyiidae) associated with Eugenia uniflora and Psidium cattleianum (Myrtaceae). Iheringia Ser Zool 101(1-2):69-74.

MAIA, V.C. \& OLIVEIRA, J.C. 2010. Galhas de insetos da Reserva Biológica Estadual da Praia do Sul (Ilha Grande, Angra dos Reis, RJ). Biota Neotropic 10(4):227-238. http://www.biotaneotropica.org.br/v10n4/pt/abstract?inven tory+bn04110042010

MAIA, V. C. \& TAVARES, M. T. 2000. Cordiamyia globosa Maia (Diptera, Cecidomyiidae), flutuação populacional e parasitoides associados. Rev. Bras. Zool. 17(3): 589-593.

MAIA, V. C.,AZEVEDO, M. A. P. \& COURI, M. S. 2002. New contribution to the knowledge of the gall midges (Diptera, Cecidomyiidae) from the restinga of Barra de Maricá (Rio de Janeiro, Brazil). Studia Dipt. 9(2): 447-452.

MAIA, V.C., CONSTANTINO, P.A.L. \& MONTEIRO, R.F. 2005. New gall midges (Diptera, Cecidomyiidae) associated with two species of Eugenia (Myrtaceae). Rev. bras. Entomol. 49(3): 347-352.

MAIA, V. C., MAGENTA, M.A.G. \& MARTINS, S. E. 2008. Ocorrência e Caracterização de Galhas de Insetos em Áreas de Restinga ee Bertioga (São Paulo, Brasil). Biota Neotropic 8 (1): 167-197. http://www.biotaneotropica. org.br/v8n1/en/abstract?inventory+bn02408012008 
MARTINS, L. R., SAMPAIO, P. S. P. \& MAGENTA, M. A. G. 2008 Caracterização florística de comunidades vegetais de restinga em Bertioga, SP, Brasil Acta bot. bras. 22(1): 249-274.

MONTEIRO, R.F., FERRAZ, F.F.F., MAIA, V.C. \& AZEVEDO, M.A.P DE 1994. Galhas entomógenas em restingas: uma abordagem preliminar. Anais da Academia de Ciências de São Paulo - ACIESP, 3(87): 210-220.

MONTEIRO, R.F., ODA, R.A.M., NARAHARA, K.L. \& CONSTANTINO, P. de A.L. 2004. Galls: Diversidade, Especificidade e Distribuição. In Pesquisa de Longa Duração na Restinga de Jurubatiba: Ecologia, História Natural e Conservação. Rocha, C.F.D. da; F. de A. Esteves \& F.R. Scarano (Orgs.). RiMa Editora: 127-141.

NEIMAN, Z. 1989. Era verde?: ecossistemas brasileiros ameaçados (10th ed.). Atual, São Paulo, Brazil.

OLIVEIRA, J. C. DE \& MAIA, V. C. 2005. Ocorrência e caracterização de galhas de insetos na restinga de Grumari (Rio de Janeiro, RJ, Brasil). Arq Mus Nac 63 (4):669-676.

RIZZINI, C.T. 1992. Tratado de fitogeografia do Brasil: aspectos ecológicos e florísticos. Âmbito Cultural Edições, São Paulo.

RODRIGUES, A. R.; MAIA, V. C. \& COURI, M. S. 2014. Insect galls of restinga areas of Ilha da Marambaia, Rio de Janeiro, Brazil. Rev Bras Entomol 58(2):173-197.

ROSARIO, A. S., SECCO, R.C., AMARAL, D. D., SANTOS, J. U. M. \& BASTOS, M.N.C, 2005. Flórula fanerogâmica das restingas do Estado do Pará. Ilhas do Algodoal e Maiandeua. Bol. Mus. Par. Emílio Goeldi. Sér. Ciências Naturais 1(3):31-48.

SANTOS, J. C., ALMEIDA-CORTEZ, J. S. \& FERNANDES, GW. 2011. Diversity of gall-inducing insects in the high altitude wetland forests in Pernambuco, Northeastern Brazil. Braz. J. Biol. 71(1):47-56.
SCARANO, F. R., DUARTE, H. M., RIBEIRO, K. T., RODRIGUES, P. J. F. P., BARCELLOS, E. M. B., FRANCO, A. C., BRULFERT, J., DELÉENS, E. \& LÜTTGE, U. 2001. Four sites with contrasting environmental stress in southeastern Brazil: relations of species, life form diversity, and geographical distribution to ecophysiological parameters. Bot J Linn Soc 136:345-364.

SILVA, L.O. \& MAIA, V. C. 2016. Insect galls of restinga da Marambaia (Barra de Guaratiba, Rio de Janeiro, RJ). Braz J Biol 76(3): 787-795. Acta bot. bras. 3(2): 253-272.

SILVA, L.O. \& RODRIGUES, A. R. 2011 Galhas de inseto do Parque Municipal da Boca da Barra, Cabo Frio (R.J.). X Congresso de Ecologia do Brasil (on line), SEB: $1-2$.

SOBRAL, M., PROENÇA, C., SOUZA, M., MAZINE, F. \& LUCAS, E. 2018. Myrtaceae. In: Lista de Espécies da Flora do Brasil. Jardim Botânico do Rio de Janeiro. Available from: http://floradobrasil.jbrj.gov.br/jabot/listaBrasil/ PrincipalUC/PrincipalUC.do (accessed 14 August 2018)

SOUZA, M. C. \& MORIM, M. P. 2008. Subtribos Eugeniinae O. Berg e Myrtinae O. Berg (Myrtaceae) na Restinga da Marambaia, RJ, Brasill. Acta bot. bras. 22(3):652-683.

VANIN, S. A. 2008. A new species of Pacholenus Schoenherr from southeastern Brazil (Coleoptera, Curculionidae, Molytinae), and new occurrences of species of the genus. Pap Avulsos de Zool 48(30):345-351.

VITORINO, M. D., PEDROSA-MACEDO, J. H. \& SMITH, C. W. 2000. The Biology of Tectococcus ovatus Hempel (Heteroptera: Eriococcidae) and its Potential as a Biocontrol Agent of Psidium cattleianum (Myrtaceae). Proceedings of the X International Symposium on Biological Control of Weeds, Montana State University, Bozeman, Montana, USA Neal R. Spencer [ed.]. pp. 651-657. 